\title{
Employing Severity of Injury to Contextualize Complex Risk Mitigation Scenarios
}

\author{
Luiz Alberto SERAFIM GUARDINI ${ }^{1,2,3}$, Anne SPALANZANI ${ }^{1}$, Christian LAUGIER ${ }^{1}$, \\ Philippe MARTINET ${ }^{2}$, Anh-Lam DO ${ }^{3}$, Thierry HERMITTE ${ }^{3}$
}

\begin{abstract}
Risk mitigation is an important element to consider in risk evaluation. Safety features have helped to decrease the death ratio over the years. However, to date, each driver assistance system works on a single domain of operation. The problem remains in how to use perception to contextualize the scene to fully minimize the collision severity in a complex emergency scenario. Up to now, works on cost maps have consider simple contextualized object in mitigation scenarios. For instance, the use of binary allowed/forbidden zones or, a fixed weight to each type of object in the scene. Our work employs the risk of injury issued by accidentology to each class of object present in the scene. Each class of object presents an injury probability with respect to the impact speed and ethical/economical/political factors. The method generates a cost map containing a collision probability along with to the risk of injury. It dynamically contextualizes the objects, since the risk of injury depends on the characteristics of the scene. Simulation and dataset results validate that changing the referred parameters alters the context and evaluation of the scene. Then, the proposed method allows a better assessment of the surroundings by creating a dynamic navigation cost map for complex scenarios.
\end{abstract}

\section{INTRODUCTION}

According to the European Commission of road safety [1], in 2018, road fatalities rated in average 49 deaths per million inhabitants in the European Union (EU). This rate had a decrease of $21 \%$ if compared to the year of 2010 . This, in large, due to the technological passive and active safety improvements on road vehicles.

Passive safety features do not work until needed: sit belts, airbags and the vehicle construction itself that helps to absorb the energy of the impact. On the other hand, active safety systems are constantly monitoring the environment and can act to prevent an accident. They fall under the Advanced Driver-Assistance Systems (ADAS) technologies.

ADAS technologies include systems such as Active Braking System (ABS), Forward Collision Warning (FCW) and Collision Avoidance (CA). The latter is one of the most complex systems developed to assure safety. It perceives technologies such as Advanced Emergency Braking (AEB) and Autonomous Emergency Steering (AES) System.

Nevertheless, each ADAS technology works independently. There is no intelligent system capable of selecting the best solution in the case of an emergency trajectory. Hence,

${ }^{1}$ INRIA Grenoble Rhone-Alpes , Chroma Team, 655 Avenue de 1'Europe, 38330 Montbonnot-Saint-Martin, name. surmaned inria.com

${ }^{2}$ INRIA Sophia Antipolis-Mditerrane, Chorale Team, 2004 Route des Lucioles, 06902 Valbonne, name. surmanedinria.com

${ }^{3}$ Renault S.A.S, 1 av. du Golf, 78288 Guyancourt, France. name.surmane@renault.com each system has its domain of operation. For instance, if a pedestrian crosses in front of a vehicle, if its speed is up to $50 \mathrm{~km} / \mathrm{h}$, AEB will be activated. Otherwise, AES will be triggered.

The problem that remains is how to rate each complex emergency scenario to contemplate the best possible solutions. For starters, one needs to evaluate the available information in the scene to determine vehicle and surroundings safety. The classical notion of safety uses the concept of collision risk between the ego vehicle an a given object in the scene.

Now, let us imagine a scenario where three objects (A, B and C) are in the reachable state of the vehicle, as shown in Figure 1. For the given scenario, collision is inevitable to one of the given objects. To simplify the analysis, let us consider an analogous probability of collision between ego and each object. Thus, the risk of collision is seemly the same for all three objects. However, each object can induce or is liable to more injuries. Therefore, paths for A, B and C will have different outcomes in terms of safety.

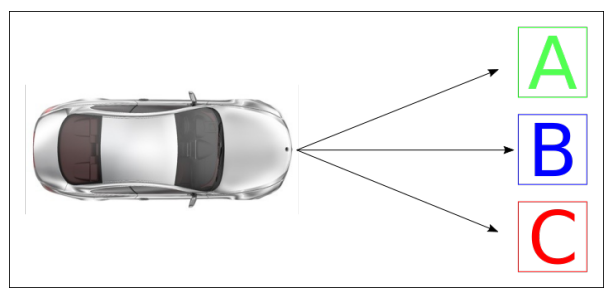

Fig. 1: Possible paths for crash mitigation.

Risk mitigation techniques are used in such scenarios. Mitigation is a notion that has been introduced with the advent of active safety systems. It allows us to deal with scenarios in which collision is unavoidable, intending to minimize crash severity to preserve the human life. The goal is then to determine the path with least danger.

To date, risk mitigation focuses on risk evaluation in terms of minimizing the impact velocity. Besides, current risk mitigation methods lack distinction of objects when dealing with more complex scenarios. Moreover, those works only consider the severity related to the occupants of the ego vehicle, disregarding the effects of a collision to other objects in the scene. For instance, a pedestrian should be avoided due to possible vehicle instability during collision [2].

Our work focus on the development of a probabilistic cost map that expresses the Probability of Collision with Injury Risk (PCIR). On top of the environment perception, 
it includes the severity of injury in the event of a collision between ego and the objects in the scene. This cost map provides enhanced information to perform vehicle motion planning in emergency complex scenarios. To the best of our knowledge there are no results in the literature on the use of severity of injury serving as a basis for decision-making.

Simulation and dataset results show that the scene interpretation does change when employing probability of injury. The same scene set with different objects (cars, pedestrians, cyclists etc) is assessed differently for different parameters. These parameters include vehicle and object velocities, as well as ethical, economical and political criteria.

The remaining of the paper is organized as follows. Section II proposes an overview on the work related to risk and crash mitigation. Section III presents the proposed probabilistic risk map system. Section IV shows the methodology for the development of the probabilistic cost map method proposed by this work. Section V presents the results and discussions. Section VI provides conclusion remarks.

\section{RELATED WORK}

Table I brings a comparison between previously developed risk mitigation methods and the one presented on this work. The severity criterion identifies if the given method considers severity of injury (i.e. slight, severe, death) when a collision between ego and an obstacle happens. Risk assessment criterion establishes if risk evaluation is considered on the method. Dynamic objects is checked if the method works with dynamic objects in the scene rather than just static ones. Data uncertainty expresses the presence of noise in data acquisition or a deterministic scenario otherwise. Object semantics points if the objects in the scene are classified and if there is more than one type of object in the scene (e.g. car, pedestrian, cyclist etc). The sign "-_" represents a subject lightly considered, whereas "+" represents a subject deeply considered on the related work.

TABLE I: Work comparison

\begin{tabular}{|l|c|c|c|c|c|}
\hline & Severity & $\begin{array}{c}\text { Risk } \\
\text { assessment }\end{array}$ & $\begin{array}{c}\text { Dynamic } \\
\text { Objects }\end{array}$ & $\begin{array}{c}\text { Data } \\
\text { Uncertainty }\end{array}$ & $\begin{array}{c}\text { Object } \\
\text { Semantics }\end{array}$ \\
\hline Beal and Giugliano [3] & - & + & + & - & - \\
\hline Arikere et al [4] & - & + & + & - & - \\
\hline Gallen et al [5] & ++ & - & + & - & - \\
\hline Wang et al [2] & + & ++ & + & - & + \\
\hline Lee and Kum [6] & + & ++ & + & - & + \\
\hline \hline Proposed method & ++ & ++ & + & ++ & + \\
\hline
\end{tabular}

Giugliano and Beal [3] work focused on developing a methodology to minimize collision velocity for a three cars pileup. The ego is between a stationary leading car and a tailing striking car. The set up makes collision is unavoidable but brings the possibility of finding the optimal velocity to minimize the energy of impact. It is a 1 degree of freedom (DOF) scenario that only allows longitudinal movement (no lateral maneuvering is permitted). Nonetheless, there is no distinction for frontal and rear collision. The scenario only considers vehicle-to-vehicle collision.

Arikere et al [4] developed an integrated controller that performs both longitudinal acceleration and yaw stability control. The aim is to better balance the oncoming vehicle collision risk against loss of control. The method considers severity as a function of velocity. The scenario only considers vehicle-to-vehicle collision.

Gallen et al [5] performs a top-down approach to modulate the reference speed in adverse conditions. Severities were classified as slight, serious and fatal, established by [7], using the relationship between speed and car driving injury. A reference safe velocity for the intended severity when considering adverse conditions such as rain or fog. The work, however, does not intend to minimize the severity, only to equalize it to initial conditions as for human drivers (reaction time and driving characteristics).

Wang et al [2] proposes to find a path with minimal severity when crash is inevitable. Obstacles and road boundaries are constrained by using artificial potential fields method. The latter is a function of overlap angle, relative impact speed and mass ratio between ego vehicle and object. A model predictive control (MPC) is developed to accommodate the cost function as well as the constraints. This MPC is used to compute the severity related to the potential crash energy.

Lee and Kum [6] presents a defensive driving methodology. When faced with dangerous situations, a collision avoidance/mitigation system (CAMS) is deployed. It evaluates the risks associated with all surrounding vehicles. The goal is to maneuver the ego vehicle into the safer region when possible. A Predictive Occupancy Map (POM) is developed to simultaneously identify potential multi-vehicle risks in spatial and temporal space. The risk is classified as the inverse of the time to simultaneous occupancy among ego and objects in the scene.

It is also possible to find risk mitigation methods in patents. The risk associated with one or more threats that may collide with vehicle is presented in [8] for semi-autonomous vehicles, and in [9] and [10] for autonomous vehicles. The mitigation of risk for an autonomous vehicle relative to turning objects is presented in [11]. They deal with the mitigation risk but do not classify objects and only consider the severity for the ego vehicle occupants.

Thus, some criteria developed in our work differ from the ones previously developed. First, our proposed method considers a probabilistic environment. Hence, characteristics of objects in the scene are not deterministic. Data carries uncertainty due to, for instance, sensor physical limitations and occlusion. This plays a major role when developing the cost map.

Second, the probabilistic grid representation allows a multi-object risk assessment. It is possible to analyze the scene closer to a human point of view, since many objects can be taken into consideration. Besides, each object has a semantic information, meaning that it is possible to distinguish pedestrian, cyclists, cars and trucks. This allows to work on complex scenarios containing several different objects.

Third, working with severity of injury to the corresponding object is made possible once object semantics is employed. For instance, a collision to a pedestrian at $30 \mathrm{~km} / \mathrm{h}$ brings a different probability of injury than a collision to a car at the 
same speed. Besides, the scenario will evolve for a $90 \mathrm{~km} / \mathrm{h}$ collision considering the same agents.

Finally, our work proposes a risk assessment to all objects in the scene, including the ones related to the occupants of the ego vehicle. To present, the severity of injury is only considered to occupants of the ego vehicle. They disregard the severity and the risk associated to the object that the ego will be colliding to. It is shown that changing parameters on the scenario will change the correspondence of the severity.

\section{INPUTS FOR THE PROBABILISTIC RISK MAP}

The proposed probabilistic risk map architecture is depicted in Figure 2. It is a system that takes as inputs: (i) vehicle localization; (ii) probability of injury issued by accidentology studies; (iii) a dynamic environment representation in the form of a probabilistic occupancy grid. It generates a Probability of Collision with Injury Risk (PCIR) as output.

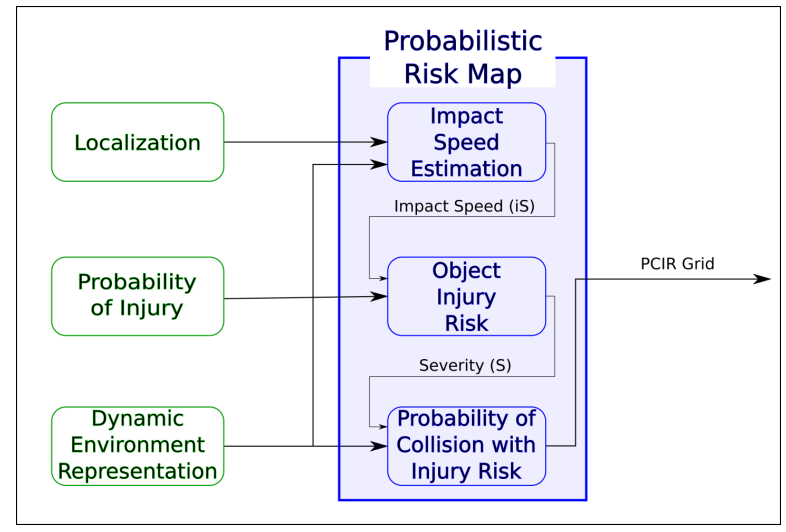

Fig. 2: Probabilistic Risk Map Architecture.

\section{A. Probability of Injury}

The probability of injury is computed from the injury curves associated to a classified object in the scene. In the scope of this work, we are interested in researches that determine the risk of injury according to the impact speed. It can be achieved by the aid of accidentology.

Accidentology is the study and analysis of the causes and effects of accidents. Though accidentology it is possible to determine the pertinent variables to contextualize the scenario. For a complete assessment it is necessary to have the probability of injury associated to all possible collision configurations: frontal, lateral, rear, rollover etc. Those configurations are connected to the type of object, since the rigidity is different, such as, for a pedestrian, another vehicle, or a wall. Improvements on the semantics on the scene will allow an even deeper classification. For instance, by determining the age of the vehicle occupants or estimating the pedestrian's, it is possible to better determine the outcome.

Among all possible injury classification, this work makes use of the Severity Curves and Maximum Abbreviated Injury Scale (MAIS) curves to establish the corresponding injuries in the case of a collision between the ego vehicle and an obstacle.
As for Severity Curves, Martin and Wu [12] performed a study that issues the probability of death for car-topedestrian frontal collision. Cuny et al [13] brings a more complete exploration by considering injury mitigation. The latter presents a set of injury risk curves, depicted in Figure 3a. It is based on French accident analysis database and divided in probability of slight (less of 24 hours or no hospitalization), severe (from 24 hours to 30 days of hospitalization) or fatal (accident related within 30 days) injuries. The statistical model is built using Akaike Information Criteria (AIC) and Bayesian Information Criterion and Bootstrap percentile methodology for its Confidence Intervals (CI). Finally, the injury probabilities were modeled using a polytomous CLOGLOG regression model.
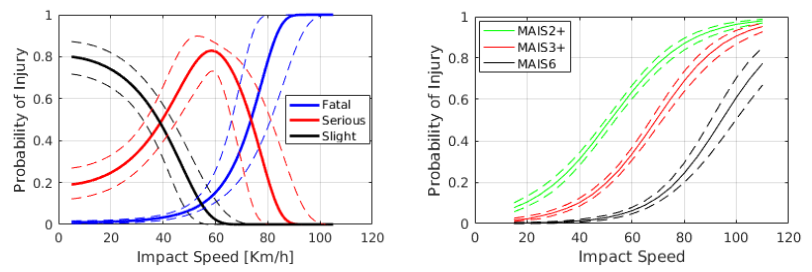

(a) Severity Curves: car-to- (b) MAIS Curves: car-to-car pedestrian frontal collision. frontal collision.

Fig. 3: Probability of Injury Curves.

The second method, the MAIS curves, is illustrated in Figure 3b. It comes from the Abbreviated Injury Scale (AIS), which integrates current medical terminology providing an internationally accepted method for rating injury severity. For this work the version 98 is used. AIS is an anatomically based, consensus derived, global severity scoring system that classifies an individual injury by body region according to its relative severity on a 6 point scale $(1=$ minor and $6=$ maximal $)$ [14], as shown in Table II. The MAIS will then represent the maximal of the AIS injuries combined. For instance, an injury is described as MAIS3+ if a casualty endures an injury with a score of 3 or higher on the AIS.

TABLE II: Abbreviated Injury Scale

\begin{tabular}{c|c|c|c} 
AIS-Code & Injury & Example & $\%$ prob. of death \\
\hline 1 & Minor & Superficial Laceration & 0 \\
\hline 2 & Moderate & Fracture sternum & $1-2$ \\
\hline 3 & Serious & Open fracture of humerus & $8-10$ \\
\hline 4 & Severe & Perforated trachea & $5-50$ \\
\hline 5 & Critical & Ruptured liver with tissue loss & $5-50$ \\
\hline 6 & Maximum & Total severance of aorta & 100 \\
\hline
\end{tabular}

One important remark is that the injury curves are data dependent. The coefficients for the logistic regression are estimated from the given database. Applying the same methodology previously described to a different database might generate different coefficients and consequently different curves.

\section{B. Dynamic Environment Representation}

One way to represent the environment is though probabilistic occupancy grids. It endures agile and robust sensor 
interpretation mechanisms and incremental discovery procedures. It also handles uncertainty thanks to probabilistic reasoning [15].

This work has made use of the Conditional Monte Carlo Dense Occupancy Tracker (CMCDOT), represented in Figure 4. It is generic spatial occupancy tracker that infers dynamics of the scene through a hybrid representation of the environment. The latter consists of static and dynamic occupancy, empty spaces and unknown areas. This differentiation enables the use of state-specific models as well as relevant confidence estimation and management of dataless areas [16].

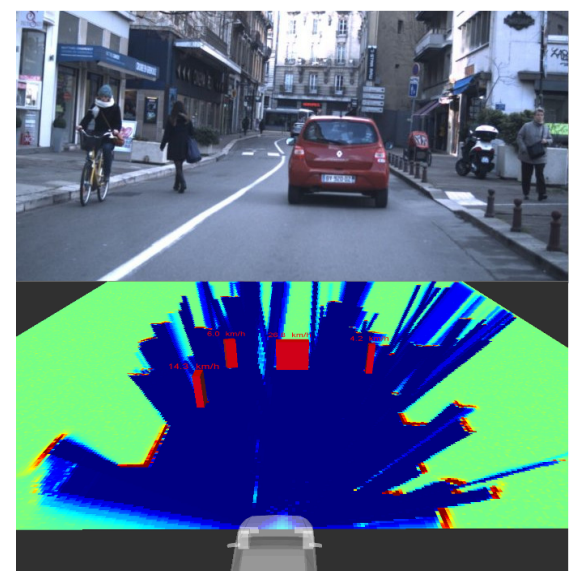

Fig. 4: CMCDOT environment representation.

Although CMCDOT occupancy grid leads to a very reliable global occupancy of the environment, it works on a subobject level, meaning that the grid by itself does not carry the information on object classification. To overcome this, Erkent et al [17] proposes a method, which estimates an occupancy grid containing detailed semantic information. The semantic characteristics include classes like road, car, pedestrian, sidewalk, building, vegetation, etc. This classification is used to assign the correct injury curve to the corresponding object.

\section{Vehicle Localization}

Localization refers to ego vehicle information, such as position, velocity, acceleration and steering angle. For simulation, a vehicle URDF model was developed by the CHROMA-IRT INRIA project team. In practice, this information is obtained by combining information from an Xsens GPS and an Inertial Measurement Unit (IMU) present in the prototype.

\section{Probability of COLlision With INJURY Risk}

This section presents the methodology for the steps of the probabilistic risk map presented in Figure 2.

\section{A. Impact Speed Estimation}

The impact speed between the ego vehicle and the elements in the scene is computed in this step. It is estimated using equation (1):

$$
i S=v_{r e l}+a \cdot T T C
$$

Where, iS stands for the impact speed, $v_{\text {rel }}$ for the relative velocity between ego and the corresponding cell, $a$ stands for the maximal acceleration, in our case, corresponds to the maximal braking force and TTC corresponds to Time-toCollision.

The relative velocity $v_{\text {rel }}$ is computed relying on the velocity information present in CMCDOT grid and vehicle odometry. A vector sum gives the desired value.

The longitudinal acceleration $a$ is estimated from a friction circle. It is limited by the lateral acceleration of the vehicle which is obtained from a lateral force linearized model [18].

Finally, the Time-To-Collision is computed using the Closest Point of Approach method [19]. It consists of an algorithm that can be applied to general two-dimensional scenarios. By computing the distance to closest approach, it is possible to determine near misses and bound sensor uncertainty. This makes it suitable for complex scenarios and situations.

\section{B. Object Injury Risk (OIR)}

The object injury risk is obtained by taking the probability of injury according to the estimate of the impact speed. If one considers simple scenarios where only one type of object is present, gathering the severity is simple, and minimizing it even simpler, since all that is needed is to minimize the impact speed. Thus, the use of injury curves only seems evident when dealing with more complex scenarios where several types of objects are present in the scene.

As a remark to what was presented in subsection III-A (Figure 3), severity curves and MAIS curves differ from each other. Whereas severity curves present each type of injury as a different curve, MAIS curves have all the information at once in one curve. Because of this, it is proposed to weight each of the probabilities of injury from the severity curves individually. For instance, if an equivalence to an MAIS3+ is desired, one can simply weight the slight injury as zero and the other curves as one, as depicted in Figure 5a.

Likewise, the idea behind our method is to assign weights to each curve that represent the injury risk for a given binary collision (car-to-car, car-to-pedestrian etc.). Those weights, though, are representative to each type of object in the scene. They must be complementary in a way that they must contextualize the desired scenario according to preferred safety criteria. For example, Figure $5 \mathrm{~b}$ presents the case where non vehicle occupants (pedestrians, cyclists) safety is essential over vehicle occupants.

Other sort of configurations are also possible. For instance, Figure 5c shows the case where the safety of the occupants of the ego vehicle is more important than others on the scene. Figure 5d displays a case where the pedestrians and cyclists takes same weight as ego vehicle occupants, but other vehicles have less priority.

As a remark, for severity curves, where there is the possibility of combining the curves, the total severity is given by equation (2).

$$
S_{\text {total }}=p D(i S) \cdot w_{p D}+p S I(i S) \cdot w_{p S I}+p S L I(i S) \cdot w_{p S L I}
$$



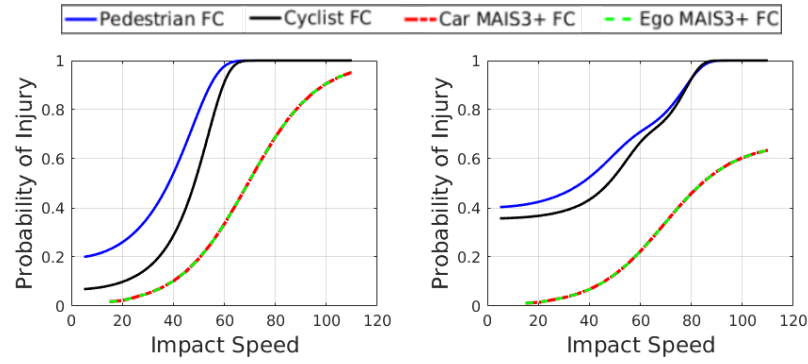

(a) Equivalence between (b) Pedestrian and Cyclist priMAIS3+ and Severity Curves. ority over vehicle occupants.
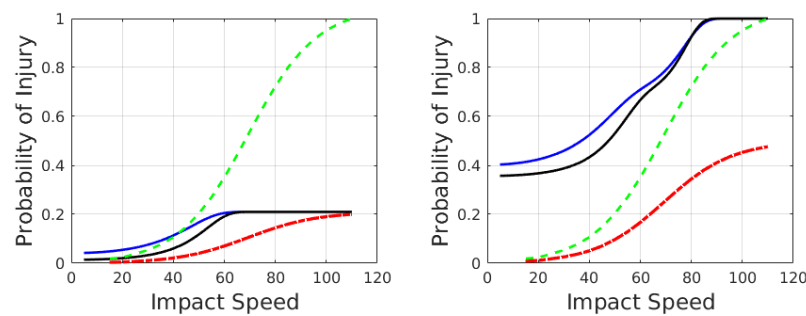

(c) Ego priority over all other (d) Ego priority over other veobjects in the scene. hicles.

Fig. 5: Different Frontal Collision (FC) severities configurations for given sets of weights.

Where $p D, p S I$ and $p S L I$ corresponds respectively to the probability of death, severe injury and slight injury with respect to the impact speed; $w_{p D}, w_{p S I}$ and $w_{p S L I}$ refer to their corresponding assigned weights.

Determining the final weights are no easy task, and it might fall into a discussion like the one from the trolley problem. Indeed, the final choice of the weights will fall under the manufacturer's responsibility and government road safety policies. To address or to propose a final set of curves is not the goal of the paper. The objective of our method is to use a final set of curves for decision making.

\section{Probability of Collision with Injury Risk (PCIR)}

Once all the elements are set, the Probability of Collision with Injury Risk is computed as shown in equation (3).

$$
P C I R=p(O c c) . S(i S, w)
$$

Where $p(O c c)$ refers to the probability of occupancy of the cell, obtained in CMCDOT grid and $S(i S, w)$ is the probability of Severity with respect to the impact speed and predefined weights.

The probability of occupancy plays a major role since it is related to the reliability of the perception. For objects closer to sensors will have a higher probability of occupancy than objects far away from the sensor. This allows us to accommodate the uncertainty of the measurement.

Once the PCIR grid is computed, all values are normalized from 0 to 1 . This way, if there is only one object in the scene, it will be treated as most critical. For more than one object, PCIR shows the relative result with respect to the most critical object.

Some assumptions are made for the probability of severity $S(i S, w)$ : (i) each vehicle has only one occupant, therefore, for a collision between ego and another vehicle, the severities are summed up. (ii) a collision between ego vehicle and pedestrian/cyclist will not bring any risk to the vehicle occupants, only to the pedestrian/cyclist.

As a disclaimer, the proposed method only computes the risk between ego-to-object collision. The risk between object-to-object collision is not considered.

\section{RESULts}

This section presents the results for computer simulation and for a dataset with real data.

\section{A. Simulation Scenario}

The scenario used for simulation is presented in Figure 6 . It is a toy example, since the presented method is not scenario dependent.

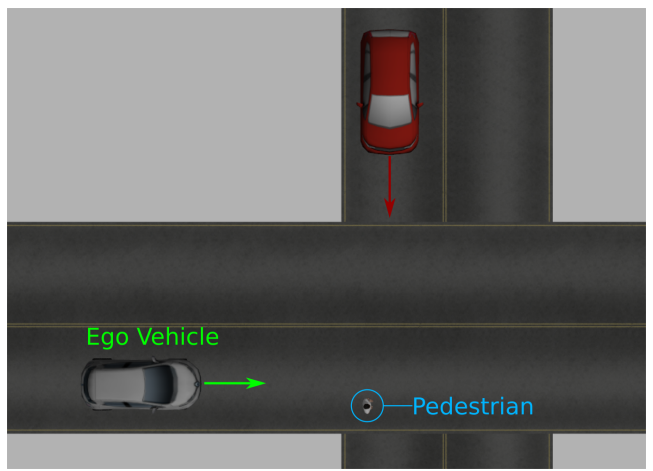

Fig. 6: Proposed simulation scenario.

It represents a crossing that can be present in cities and country roads. The ego vehicle is represented by the gray vehicle. Its speed can range between 10 to $90 \mathrm{~km} / \mathrm{h}$. The second vehicle, in red, has an unknown behavior, meaning that its braking and steering information are not known beforehand. Its speed also ranges between 10 to $90 \mathrm{~km} / \mathrm{h}$. Besides, a pedestrian (static) is present in the scene.

The aim of the simulation is to present how different parameters will change the context of the scene. Here, the considered parameters are the impact speed and the weights of the injury curves. The latter may be related to ethical, economical and political aspects. So, two different configurations are presented and its corresponding injury curves are shown in Figure 7.
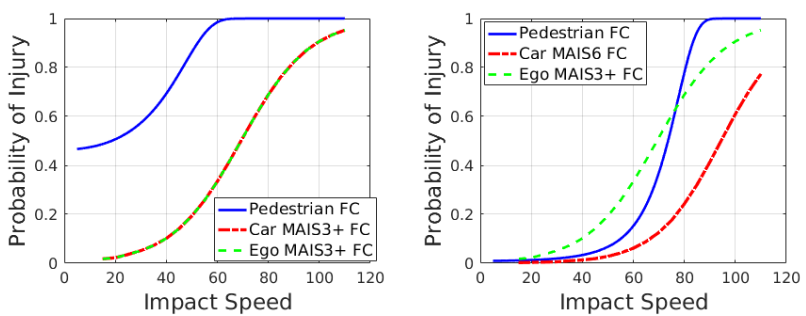

(a) Vulnerable Road Users (b) Political/economical sce(VRU) scenario configuration. nario configuration.

Fig. 7: Injury curves for the proposed scenario configurations. 
1) Vulnerable Road Users (VRU) Scenario Configuration: The first setup prioritizes vulnerable users over other vehicles. The set of curves for this arrangement is displayed on Figure 7a. All pedestrians injuries are taking into account (slight, severe and fatal), with weights of 1,3 and 3, respectively. The total probability of injury is given by equation (2). The MAIS3+ curves with weight of 3 are assigned to both ego and other vehicle.

2) Political/Economical Scenario Configuration: The second setup falls under a political/economical point of view. In this case the safety of the occupants of the ego vehicle is a greater concern with respect to others. The set of curves for this composition is displayed on Figure 7b. For this case, MAIS3+ severities are taken into account to the ego vehicle, whereas only the risk of death is considered to all other objects in the scene. The same weight is assigned to all curves.

For displaying purposes, ego vehicle longitudinal speeds of 30,60 and $90 \mathrm{~km} / \mathrm{h}$ were considered for the simulation. For the moment, no control actions were taken, since this step considers the construction and validation of the proposed Probabilistic Risk Map. The PCIR grid simulation was performed using Gazebo Simulator [20] and Rviz [21] to display the resulting grid.

\section{B. Simulation Results}

The two metrics are analyzed in the results are the influence the impact speed, and, the injury curves weights based on ethical, economical and political criteria. The influence of the impact speed is given by each row, whereas the influence of the the set of weights is observable on each column of the given figures.

The intention of the simulation is to validate the PCIR with respect to the impact speed. Thus, no braking force was considered for the simulation. Figure 8a brings a scenario where no injury curves are considered. Random weights were assigned to each type of object considering the pedestrian as more vulnerable with respect to the car. Figures $8 \mathrm{~b}$ and $8 \mathrm{c}$ depicts the results for the configurations described above.

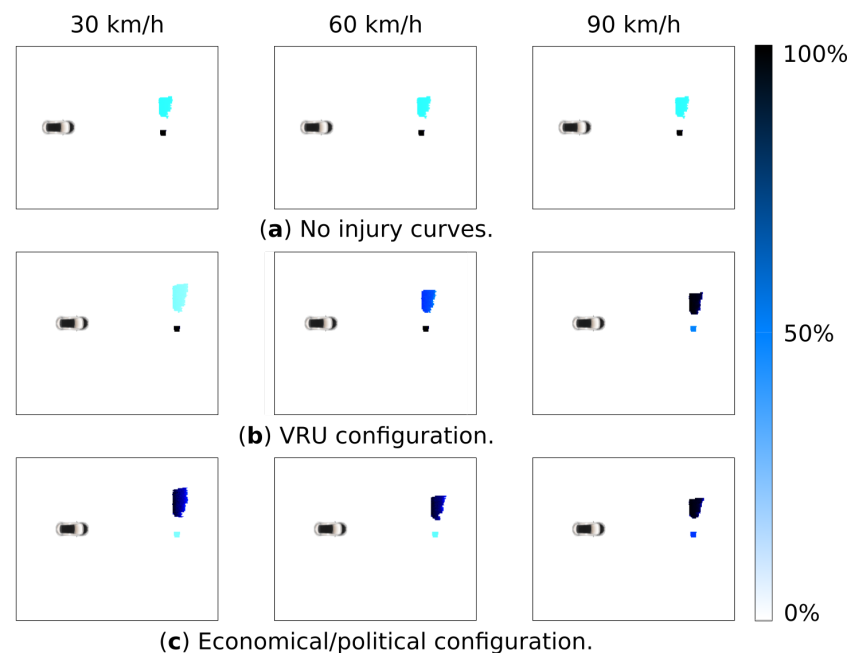

Fig. 8: Probability of Collision with Injury Risk in simulation.
From the given results, the first outcome is that the context of the scenario is too complex to consider static weights to the objects in the scene. By changing the impact speed between ego and object one can have a completely different assessment of the scene. For instance, in Figure 8, when comparing the framework at 30 and at $90 \mathrm{~km} / \mathrm{h}$. At $30 \mathrm{~km} / \mathrm{h}$ the pedestrian takes precedence, since its probability of injury is higher. On the other hand, at $90 \mathrm{~km} / \mathrm{h}$, the collision between ego and another vehicle will have a higher probability of injury compared to the collision to a pedestrian.

The second outcome is related to the ethical, economical and political influence on choosing the weights on the curves. One can notice that for both configurations, a different set of weights will also change the scene evaluation for the corresponding the impact speeds.

\section{Dataset Results}

An experiment was performed using real data from KITTI raw dataset [22]. The dataset contains object identification for vehicles (car, van, tram etc.), pedestrians and cyclists. Hence, the grid will present the probability of collision with injury risk only for those elements. Static obstacles, such as walls and light poles are not considered for the time being. The scenario is presented in Figure 9. The captured frame contains a cyclist, a car and a truck.

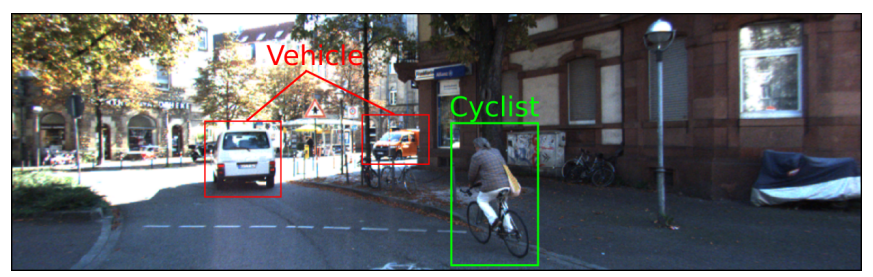

Fig. 9: KITTI dataset scenario.

The same two configurations for VRU and for economical/political weight setup were considered. The results are depicted in Figures 10a and 10b respectively.

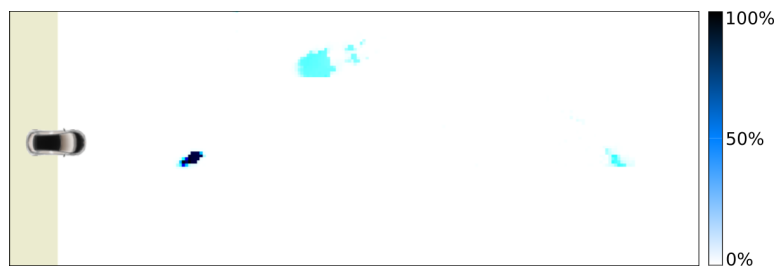

(a) VRU configuration

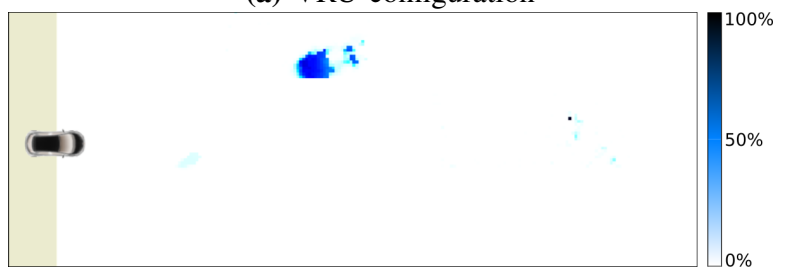

(b) Economical/political configuration.

Fig. 10: Probability of Collision with Injury Risk for KITTI dataset. 
The objective of this experiment is to validate the use of injury curves on real scenarios. One can observe a similar outcome with respect to the simulation. Changing the weights according to the intended ethical/economical/political parameters will change the scene evaluation.

One limitation of this work is related to the impact velocity estimation. For now, only constant velocities are considered. As the system works on a closed-loop, the influence of the acceleration might be dealt with by increasing the uncertainty area close to an object.

Another limitation is related to the object injury risk. For now, only frontal collision is considered. Although, it is known that lateral vehicle-to-vehicle collisions have a different probability of injury than frontal collision. The same is valid for rear vehicle-to-vehicle collision. This limitation is due to the perception in our probabilistic environment which works on a sub-object level. Forthcoming work will include the discrimination for the collision type.

\section{CONCLUSIONS}

Risk mitigation is an essential component for decision and motion planning for complex emergency scenarios. By introducing injury curves, it is possible to better contextualize a scene containing different types of objects, e.g. pedestrians, cars, cyclists. This work employed such curves and observed the influence of the impact velocity and ethical/economical/political aspects for scene evaluation. Simulation and dataset results show that the risk assessment for the setting changes according to those different parameters. Then, the proposed method aids in the scene contextualization for a better decision-making based on minimizing the risk of injury.

Future developments include the ability of dealing with non-constant velocities. It is essential, since during an emergency situation the driver or the assistance system might start braking the vehicle. Future developments also include perceive the type of collision (i.e. frontal, lateral, rear) as well as the overlap between vehicles. This is important because each type of collision contains a different set of injury curves. Some other aspects may be included in order to assess the risk, such as pedestrian and driver behavior and different types of vehicles, such as trucks and vans. Further development includes a reachability set, i.e. determine all possible reachable states of the ego vehicle in a 3 seconds window. This will allow to select the most comprehensive trajectory for collision avoidance/mitigation, while considering all possible contextualized reachable scenarios.

\section{ACKNOWLEDGEMENTS}

This work is supported by a CIFRE fellowship from Renault S.A.S. We thank the LAB (Laboratoire dAccidentologie, de Biomcanique et du comportement du conducteur) for providing the risk of injury curves issued from their accident data analysis.

\section{REFERENCES}

[1] European Comission. (2019) 2018 road safety statistics: what is behind the figures? [Online]. Available: https://ec.europa.eu/ commission/presscorner/detail/en/MEMO_19_1990

[2] H. Wang, Y. Huang, A. Khajepour, Y. Zhang, Y. Rasekhipour, and D. Cao, "Crash mitigation in motion planning for autonomous vehicles," IEEE Transactions on Intelligent Transportation Systems, vol. 20, no. 9, pp. 3313-3323, Sep. 2019.

[3] L. Giugliano and C. E. Beal, "Dynamic rear-end collision mitigation for a vehicle about to be struck," in ASME 2015 Dynamic Systems and Control Conference. American Society of Mechanical Engineers, 10 2015. [Online]. Available: https://doi.org/10.1115/dscc2015-9674

[4] A. Arikere, D. Yang, M. Klomp, and M. Lidberg, "Integrated evasive manoeuvre assist for collision mitigation with oncoming vehicles," Vehicle System Dynamics, vol. 56, no. 10, pp. 1577-1603, 2018.

[5] R. Gallen, N. Hautière, A. Cord, and S. Glaser, "Supporting drivers in keeping safe speed in adverse weather conditions by mitigating the risk level," IEEE Transactions on Intelligent Transportation Systems, vol. 14, pp. 1558-1571, 2013.

[6] K. Lee and D. Kum, "Collision avoidance/mitigation system: Motion planning of autonomous vehicle via predictive occupancy map," IEEE Access, vol. 7, pp. 52 846-52 857, 2019.

[7] D. P. Wood, N. Veyrat, C. Simms, and C. Glynn, "Limits for survivability in frontal collisions: Theory and real-life data combined," Accident Analysis and Prevention, vol. 39, no. 4, pp. 679 - 687, 2007.

[8] Y.-T. Dai, "Vehicle hazard detection and warning system," Patent US10 246 065B2, 2016.

[9] D. V. Prokhorov and V. Vladimerou, "Risk mitigation for autonomous vehicles relative to oncoming objects," Patent US9 573 592B2, 2014.

[10] B. BEAUVAIS and S. J. Lauffer, "Vehicle collision severity mitigation," Patent US10 246 090B2, 2016.

[11] D. V. Prokhorov and V. Vladimerou, "Risk mitigation for autonomous vehicles relative to turning objects," Patent US9 701 306B2, 2014.

[12] J.-L. Martin and D. Wu, "Pedestrian fatality and impact speed squared: Cloglog modeling from french national data," Traffic Injury Prevention, vol. 19, 052017.

[13] S. Cuny, E. Lécuyer, T. Hermitte, N. Bertholon, and H. Chajmowicz, "A tool to assess pedestrian safety: Risk curves by injury severity and their confidence intervals for cartopedestrian front collision," in IRCOBI 2018.

[14] "Association for the Advancement of Automotive Medicine: The Abbreviated Injury Scale 1990 Revision - Update 98. 1998. Barrington, IL: Association for the Advancement of Automotive Medicine."

[15] A. Elfes, "Using occupancy grids for mobile robot perception and navigation," Institute of Electrical and Electronics Engineers (IEEE), vol. 22, no. 6, pp. 46-57, June 1989.

[16] L. Rummelhard, J. Lussereau, J.-A. David, C. Laugier, S. Dominguez, G. Garcia, and P. Martinet, "Perception and Automation for Intelligent Mobility in Dynamic Environments," in ICRA 2017 Workshop on Robotics and Vehicular Technologies for Self-driving cars, 062017. [Online]. Available: https://hal.inria.fr/hal-01592566

[17] Ö. Erkent, C. Wolf, C. Laugier, D. Sierra González, and V. R. Cano, "Semantic Grid Estimation with a Hybrid Bayesian and Deep Neural Network Approach," in IROS 2018 - IEEE/RSJ International Conference on Intelligent Robots and Systems. Madrid, Spain: IEEE, 10 2018, pp. 1-8. [Online]. Available: https://hal.inria.fr/hal-01881377

[18] J. Y. Wong, Theory of Ground Vehicles. John Wiley \& Sons, Inc, 2001.

[19] C. Schwarz, "On computing time-to-collision for automation scenarios," Transportation Research Part F: Traffic Psychology and Behaviour, vol. 27, pp. 283 - 294, 2014, vehicle Automation and Driver Behaviour.

[20] N. Koenig and A. Howard, "Design and use paradigms for gazebo, an open-source multi-robot simulator," in 2004 IEEE/RSJ International Conference on Intelligent Robots and Systems (IROS) (IEEE Cat. No.04CH37566), vol. 3, Sep. 2004, pp. 2149-2154 vol.3.

[21] M. Quigley, B. Gerkey, K. Conley, J. Faust, T. Foote, J. Leibs, E. Berger, R. Wheeler, and A. Ng, "Ros: an open-source robot operating system," in Proc. of the IEEE Intl. Conf. on Robotics and Automation (ICRA) Workshop on Open Source Robotics, may 2009.

[22] A. Geiger, P. Lenz, C. Stiller, and R. Urtasun, "Vision meets robotics: The kitti dataset," The International Journal of Robotics Research, vol. 32, no. 11, pp. 1231-1237, 2013. [Online]. Available: https://doi.org/10.1177/0278364913491297 